\title{
EXTENDING MONOTONE DECOMPOSITIONS OF 3-MANIFOLDS
}

\author{
BY \\ R. H. BING( ${ }^{(1)}$
}

1. Introduction. Suppose $X$ is a closed subset of Euclidean 3-space $E^{3}$ and $f$ is a compact monotone map of $X$. We shall be concerned with conditions under which $f$ may be extended to a compact monotone map of $E^{3}$ such that the image space is topologically $E^{3}$.

It was our original intention to show that if $f$ has only a countable number of point inverses and none of these separates $E^{3}$, then there is a monotone upper semicontinuous decomposition of $E^{3}$ onto itself with the point inverses being elements of the decomposition. As we developed tools for proving this, we found that these tools proved more. Since this paper was written we learn that Ralph J. Bean studies similar situations in a paper entitled "Repairing embeddings and decompositions in $S^{3}$ " to appear in Duke Mathematical Journal.

We find it convenient to state our preliminary results for compact 3-manifolds, so in first sections of this paper we deal with the 3-sphere $S^{3}$ rather than $E^{3}$. We suppose that $E^{3}$ and $S^{3}$ have their customary rectilinear structures.

By an $n$-manifold we mean a separable metric space which is locally like Euclidean $n$-space. We do not imply without specification that it is either connected, compact, triangulated, or orientable. However, we do imply that it is without boundary. If we want to permit the possibility of a boundary, we call it a manifold with boundary. It is called a manifold with nonnull boundary if we want to say that it has a boundary for certain.

For a manifold with nonnull boundary (such as a ball, arc, disk), we use Bd $M$ to denote the boundary of $M$ (set of points which have open neighborhoods homeomorphic to closed Euclidean half space) and Int $M$ to denote the interior of $M$ (set of points with open neighborhoods homeomorphic to Euclidean space).

When we call a manifold or geometric object triangulated, we mean that a particular triangulation is assigned to it. If no such assignment has been made but the object is isometric to some geometric complex, we say that the object is polyhedral. If the object is topologically equivalent to some geometric complex, we say that it is triangulable or can be triangulated. For example, in the plane, a polygon is regarded as triangulated and the closed disk bounded by the polygon is polyhedral. If a particular triangulation were assigned to the polygonal disk, it would

Received by the editors December 18, 1968.

( ${ }^{1}$ Paper partially written while a visitor at Australian National University with support from Contract NSF-3857 and University of Wisconsin. Completed under ONR Contract.

Copyright (C) 1970, American Mathematical Society 
then be triangulated. A round disk would be neither triangulated nor polyhedral, but it would be triangulable.

Since it is known ([3], [4], [8], [9]) that any 3-manifold with boundary is triangulable, it does not restrict those being studied to limit our consideration to those that are polyhedral. By using the polyhedral requirement we are able to specify that certain of our maps are piecewise linear and certain subsets are polyhedral.

A 2-manifold is orientable if it contains no Möbius band; a 3-manifold is orientable if it contains no solid Klein bottle. A solid Klein bottle is a set topologically equivalent to the cartesian product of a Möbius band and a segment.

A closed polyhedral object is called a polyhedron. We do not insist that it it be compact. A polyhedral $n$-cell is a polyhedron that can be given a triangulation compatible with its metric which is isomorphic to some rectilinear triangulation of the ordinary $n$-cell. A compact 1-dimensional polyhedron is called a polyhedral 1-complex.

We say that there is an elementary geometric collapse of a polyhedron $P_{1}$ onto a subpolyhedron $P_{2}$ if for some integer $m$ there is a polyhedral $m$-cell $B^{m}$ such that $P_{1}=P_{2} \cup B^{m}$ and $P_{2} \cap B^{m}$ is a polyhedral $(n-1)$-cell on $\mathrm{Bd} B^{m}$. We write $P_{1} \searrow P_{2}$. We say that $P$ collapses to $Q$ if there is a finite sequence of elementary collapses such that

$$
P=P_{1} \searrow P_{2} \searrow \cdots \searrow P_{j}=Q
$$

Suppose $P$ is a compact polyhedron in a polyhedral $n$-manifold with boundary $M^{n}$. We say that $N^{n}$ is a regular neighborhood of $P$ in $M^{n}$ if $N^{n}$ is an $n$-manifold with boundary in $M^{n}, N^{n}$ collapses to $P$, and $P$ lies in an open subset of $M^{n}$ in $N^{n}$. It is known ([7], [10], [12]) that if $N_{1}^{n}, N_{2}^{n}$ are regular neighborhoods of $P$, then there is a piecewise linear homeomorphism of $N_{1}^{n}$ onto $N_{2}^{n}$.

We call an $i$-dimensional polyhedron $P^{i}$ an $i$-spine of a compact polyhedral $n$-manifold with boundary $M^{n}$ if $M^{n}$ collapses to $P^{i}$ and $P^{i}$ does not collapse onto any proper subpolyhedron.

A collection $G$ of mutually exclusive compact sets in a metric space $X$ is called an upper semicontinuous collection if $X$ is the union of elements of $G$ and for each open set $U$ in $X$ containing an element of $G$, the union of elements of $G$ in $U$ is open. The decomposition space $X / G$ has as points the elements of $G$ and as open sets collections of these "points" whose union is open in $X$. The decomposition map of $X$ onto $X / G$ is the one which sends the point $x \in X$ into the "point" $g_{X} \in X / G$ which contains it (when $g_{X}$ is regarded as a set in $X$ ). The decomposition map may alternatively be called an upper semicontinuous decomposition or for brevity merely a decomposition. We speak of the decomposition of $X$ as being into sets (elements of $G$ ) and onto $X / G$. The decomposition is monotone if each element of $G$ is connected.

If $A$ is a compact subset of $X$ rather than an upper semicontinuous decomposi- 
tion of $X$, we use $X / A$ to denote $X / G_{A}$ where $G_{A}$ is the upper semicontinuous decomposition of $X$ whose only nondegenerate element is $A$.

Closely related to the notion of a monotone upper semicontinuous decomposition is that of a monotone mapping. A continuous transformation is called a map or mapping. A map $f$ of $X$ is monotone if the point inverses are compact continuathat is, for each $y \in f(X), f^{-1}(y)$ is compact and connected.

If $f$ is a monotone map of a space $X$ onto a space $Y$ such that the point inverses form an upper semicontinuous collection $G$, consideration of the 1-1 continuous map of $[0, \infty)$ ontu a circle shows that one cannot conclude that $Y$ is homeomorphic to $X / G$. However, they are homeomorphic if the map $f$ is compact. A mapping is compact if the inverses of compact sets are compact. See [11] for a discussion of relationships between decompositions and mappings.

A map $f$ of a polyhedron $P_{1}$ into a polyhedron $P_{2}$ is piecewise linear if $P_{1}, P_{2}$ have triangulations $T_{1}, T_{2}$ compatible with the metrics of $P_{1}, P_{2}$ so that $f$ takes each simplex of $T_{1}$ linearly into a simplex of $P_{2}$. In case $P_{1}$ is compact we can say that $f$ takes each simplex of $T_{2}$ linearly onto a simplex of $T_{1}$ but for noncompact polyhedra, this is too restrictive. If $g(x)=1 / x(x \geqq 1)$ there is a piecewise linear map $f$ of $[1, \infty)$ into $[0,1]$ such that $|f(x)-g(x)| \leqq 1 / 2 x$.

Throughout this paper we shall use $M^{3}$ to denote a compact, connected, polyhedral 3-manifold with boundary. Perhaps it has no boundary but if this is required, it is so specified.

2. Boring holes in $M^{3}$. Suppose $H^{3}$ is a polyhedral 3-cell in $M^{3}$ so that $H^{3} \cap \mathrm{Bd} M^{3}$ is the union of two disjoint disks $D_{0}^{2}, D_{1}^{2}$. If we form a new 3-manifold with boundary $M_{1}^{3}$ by removing Int $H^{3} \cup$ Int $D_{0}^{2} \cup$ Int $D_{1}^{2}$ from $M^{3}$, we say that $H^{3}$ is a hole bored in $M^{3}$, and $D_{0}^{2}, D_{1}^{2}$ are the ends of the hole. We say that $M_{1}^{3}$. was obtained by boring a hole in $M^{3}$.

It is known that if $M^{3}$ has a nonnull boundary, then it can be changed into a cube with handles (some of the handles will be twisted or nonoriented if $M^{3}$ is not orientable) by boring a finite number of holes in $M^{3}$. A convenient way to find the holes is to bore them about spanning arcs in the 1-skeleton of some triangulation of $M^{3}$. Theorem 2.2 is a variation of this result. In order to prove Theorem 2.2 we first use Theorem 2.1 to build a 2-spine $Q^{2}$ which perhaps looks like 3-pages of a book at some places. Casler proved a stronger version of Theorem 2.1 in [6] where he built a $Q^{2}$ with a standard structure. We do not need this standard structure so for completeneness we include an elementary proof of Theorem 2.1 .

THEOREM 2.1. If $M^{3}$ has a nonnull boundary, there is a spine $Q^{2}$ for $M^{3}$ such that if two disks in $Q^{2}$ intersect in an interior point of each, their intersection is 2-dimensional.

Proof. Let $T$ be a triangulation of $M^{3}$. Our intention is to change $T$ to a cellular decomposition $T^{\prime}$ such that if two disks in the 2-skeleton of $T^{\prime}$ intersect in an interior point of each, their intersection is 2-dimensional. 
Blunt each 3-simplex of $T$ by removing 3-simplexes about each vertex as shown in Figure 2 on p. 17 of [1]. Each of these removed simplexes is similar to the original and has edges one-third as long. For each vertex of $T$, the union of the blunted ends containing the vertex is a 3-cell which is an element of the cellular subdivision $T^{\prime}$.

Consider each of the blunted 3-simplexes. What is left of its edges are trimmed off as shown in Figure 2 on p. 17 of [1]. The union of the prismatic pieces about the middle third of each edge is a 3-cell which is an element of the subdivision $T^{\prime}$.

The other 3-dimensional cells in $T^{\prime}$ are the blunted and trimmed 3-simplexes of $T$.

The spine $Q^{2}$ promised by Theorem 2.1 is obtained by considering the 3-cells of $T^{\prime}$ and ordering them $B_{1}^{3}, B_{2}^{3}, \ldots, B_{m}^{3}$ so that each $\mathrm{Bd} B_{i}^{3}$ contains a polyhedral 2-cell $B_{i}^{2}$ which it shares in common with either $\mathrm{Bd} M^{3}$ or some $\mathrm{Bd} B_{j}(j<i)$. Then

$$
M^{3} \searrow M^{3}-\text { Int } B_{1}^{3}-\text { Int } B_{1}^{2} \searrow \cdots \searrow M^{3}-\bigcup_{i=1}^{m} \text { Int } B_{i}^{3}-\bigcup_{i=1}^{m} \text { Int } B_{i}^{2}=Q_{1}^{2} .
$$

Then $Q^{2}$ is obtained by collapsing $Q_{1}^{2}$ as much as possible. That $M^{3}$ is a regular neighborhood of this reduced $Q^{2}$ follows from the result ([7], [10], [12]) that if one polyhedron collapses to a second, each regular neighborhood of the larger polyhedron is a regular neighborhood of the smaller. Likely $Q^{1}$ is a 2 -spine but its dimension might be less than 2 .

THEOREM 2.2. If $M^{3}$ has precisely two boundary components, it is possible to obtain a 3-manifold with boundary $M_{0}^{3}$ from $M^{3}$ by boring a finite number of holes in it such that for some polyhedral 2-manifold $M^{2}$ there is a piecewise linear homeomorphism of $M^{2} \times[0,1]$ onto $M_{0}^{3}$.

Proof. We shall show that by boring holes in $M^{3}$, it can be changed to a polyhedral 3-manifold with boundary $M_{0}^{3}$ such that $M_{0}^{3}$ has two boundary components and $M_{0}^{9}$ collapses to a 2-spine $M^{2}$ which is a 2-manifold $M^{2}$. The truth of Theorem 2.2 then follows from the facts that $M_{0}^{3}$ is a regular neighborhood of $M^{2}$, any two regular neighborhoods of $M^{2}$ are piecewise linearly homeomorphic, and there is a regular neighborhood of $M^{2}$ piecewise linearly homeomorphic to $M^{2} \times[0,1]$.

It follows from Theorem 2.1 that $M^{3}$ has a 2-spine $Q^{2}$ such that if two disks in $Q^{2}$ meet in an interior point of each, their intersection is 2-dimensional. We suppose $Q^{2}$ is such a spine that lies in Int $M^{3}$. The 2-manifold $M^{2}$ promised by the preceding paragraph is obtained by altering $Q^{2}$.

If there is a 1-cell $A_{1}$ and three disks $D_{1}^{2}, D_{2}^{2}, D_{3}^{2}$ in $Q^{2}$ such that $D_{i}^{2} \cap D_{j}^{2}=A^{1}$ $(i \neq j)$, some one of the disks is not a subset of the part of $Q^{2}$ irreducible with respect to separating the two boundary components from each other in $M^{3}$. Holes can be bored in $M^{3}$ and through this disk so as to obtain a 3-manifold with two boundary components which collapses onto a proper subpolyhedron of $Q^{2}$.

Let $M_{1}^{3}$ be a 3-manifold with two boundary components obtained by boring holes in $M^{3}$ such that $M_{1}^{3}$ contains a spine $Q_{1}^{2} \subset Q^{2}$ such that no further boring 
of holes in $M_{1}^{3}$ results in a 3-manifold with two boundary components and a spine which is a proper subset of $Q_{1}^{2}$. Note that $Q_{1}^{2}$ is the union of a 2-manifold $M_{1}^{2}$ and a 1-complex. By adjusting this 1-complex we may suppose that it is the union of a finite number of disjoint arcs $A_{1}^{1}, A_{2}^{1}, \ldots, A_{m}^{1}$ such that each $A_{i}^{1} \cap M_{1}^{2}=\operatorname{Bd} A_{i}^{1}$.

Boring holes in $M_{1}^{3}$ with the $A_{i}^{1}$ 's as centers of the holes produces a 3-manifold with boundary $M_{0}^{3}$ such that $M_{0}^{3}$ has two boundary components and a 2-manifold $M^{2}$ as a spine where $M^{2}$ is obtained from $M_{1}^{2}$ by replacing the $A_{i}^{1}$ 's and disks on $M_{1}^{2}$ about their ends by annuli.

3. Decomposing $M_{3}$ into 2-manifolds and pinched 2-manifolds. A pinched manifold results from identifying two points of the same manifold. If two objects are joined at a point, their union is called the wedge of the two objects. The wedge of two tangent round 2-spheres is an example of a pinched 2-manifold. If a cube with handles has one of its handles squeezed to a point, the boundary of the resulting object may be an example of a pinched 2-manifold.

Suppose $B^{2}$ is a boundary component of $M^{3}$. It is known (see for example Lemma 1 of [8]) that there is a piecewise linear homeomorphism $h$ of $B^{2} \times[0,1]$ into $M^{3}$ so that for each $x \in B^{2}$,

$$
h(x \times 0)=x \text { and } h\left(B^{2} \times(0,1]\right) \subset \text { Int } M^{3} .
$$

We call $h\left(B^{2} \times[0,1]\right)$ a polyhedral cartesian product neighborhood of $B^{2}$.

In the following three theorems we show that certain 3-manifolds can be decomposed into 2-manifolds and pinched 2-manifolds. A map is open if the images of open sets are open.

THEOREM 3.1. Suppose $M^{3}$ has precisely two boundary components $B_{0}^{2}, B_{1}^{2}$. Then there is a monotone piecewise linear open map $f$ of $M^{3}$ onto $[0,1]$ such that $f^{-1}(0)$ $=B_{0}^{2}, f^{-1}(1)=B_{1}^{2}$, and for each $t \in[0,1], f^{-1}(t)$ is either a 2-manifold or a pinched 2-manifold. If $[a, b] \subset[0,1]$ and for each $t \in[a, b], f^{-1}(t)$ is a 2-manifold, there is a piecewise linear homeomorphism of $f^{-1}[a, b]$ onto $f^{-1}(a) \times[a, b]$ that takes $f^{-1}(t)$ onto $f^{-1}(a) \times t$.

Proof. Let $h_{0}$ be a piecewise linear homeomorphism of $B_{0}^{2} \times[0,1 / 3]$ onto a cartesian product neighborhood of $B_{0}^{2}$ in $M^{3}$ so that $h_{0}(x \times 0)=x$ and let $h_{1}$ be a piecewise linear homeomorphism of $B_{1}^{2} \times[2 / 3,1]$ onto a cartesian product neighborhood of $B_{1}^{2}$ in $M^{3}$ so that $h_{1}(x \times 1)=x$. We suppose these two cartesian product neighborhoods do not intersect each other. Then

$$
M^{3}-h_{0}\left(B_{0}^{2} \times[0,1 / 3)\right)-h_{1}\left(B_{1}^{2} \times(2 / 3,1]\right)=M_{1}^{3}
$$

is a 3-manifold with precisely two boundary components $h_{0}\left(B_{0}^{2} \times 1 / 3\right), h_{1}\left(B_{1}^{2} \times 2 / 3\right)$.

It follows from Theorem 2.2 that it is possible to bore a finite number of mutually exclusive polyhedral holes $H_{1}^{3}, H_{2}^{3}, \ldots, H_{n}^{3}$ in $M_{1}^{3}$ such that each of these holes has both ends on the same boundary component of $M_{1}^{3}$ and for some 2-manifold $M^{2}$, there is a piecewise linear homeomorphism $h$ of $M^{2} \times[1 / 3,2 / 3]$ onto the 
closure of $\left(M_{1}^{3}-\bigcup H_{i}^{3}\right)$. We suppose $h\left(M^{2} \times 1 / 3\right), h\left(M^{2} \times 2 / 3\right)$ are the altered $h_{0}\left(B_{0}^{2} \times 1 / 3\right), h_{1}\left(B_{1}^{2} \times 2 / 3\right)$ respectively and define

$$
f\left(h\left(M^{2} \times t\right)\right)=t, \quad t \in[1 / 3,2 / 3] .
$$

Let $H_{1}^{3}, H_{2}^{3}, \ldots, H_{m}^{3}$ be the holes bored in $M_{1}^{3}$ from $h_{0}\left(B_{0}^{2} \times 1 / 3\right)$. For each $H_{i}^{3}$, let $C_{i}^{3}, C_{i}^{3 \prime}$ be caps over the ends of $H_{i}^{3}$ so that each $C_{i}^{3}$ is a polyhedral 3-cell in $h_{0}\left(B_{0}^{2} \times[0,1 / 3]\right)$ such that $C_{i}^{3} \cap h_{0}\left(B_{0}^{2} \times 1 / 3\right)$ is an end of $H_{i}^{3}, \mathrm{Bd} C_{i}^{3} \cap h_{0}\left(B_{0}^{2} \times 1 / 6\right)$ is a point and for each $t \in(1 / 6,1 / 3), \mathrm{Bd} C_{i}^{3} \cap h_{0}\left(B_{0}^{2} \times t\right)$ is a simple closed curve; the $C_{i}^{3 \prime}$ are similarly defined. We suppose that no two of the caps intersect each other.

If $t \in[0,1 / 3)$ and $p \in h_{0}\left(B_{0}^{2} \times t\right)-\bigcup$ Int $C_{i}^{3}-\bigcup$ Int $C_{i}^{3 \prime}$, we define $f(p)=t$.

For each $i=1,2, \ldots, m$, consider the polyhedral 3-cell $H_{i}^{3} \cup C_{i}^{3} \cup C_{i}^{3 \prime}$. We note that $f$ has already been defined on its boundary. Triangulate this boundary so that $f$ is linear with respect to the triangulation. Then triangulate $H_{i}^{3} \cup C_{i}^{3} \cup C_{i}^{3 \prime}$ by coning from an interior point $p_{i}$.

Let $\varepsilon_{1}, \varepsilon_{2}, \ldots, \varepsilon_{m}$ be different numbers in $(1 / 6,1 / 3)$. Define $f\left(p_{i}\right)=\varepsilon_{i}$ and extend $f$ linearly to the simplexes of the conical triangulation of $H_{i}^{3} \cup C_{i}^{3} \cup C_{i}^{3}$. Then $\left(H_{i}^{3} \cup C_{i}^{3} \cup C_{i}^{3 \prime}\right) \cap f^{-1}(t)$ is the union of two points if $t=1 / 6$, the union of two disjoint disks (cones) if $t \in\left(1 / 6, \varepsilon_{i}\right)$, the union of two cones with a common vertex $p_{i}$ if $t=\varepsilon_{i}$, and an annulus if $t \in\left(\varepsilon_{i} 1 / 3\right]$. It is to be noted that $f$ is open at $p_{i}$.

Note that for some neighborhood $\left(\varepsilon_{i}-\delta, \varepsilon_{i}+\delta\right)$ of $\varepsilon_{i}$, there is a piecewise linear projection $r_{i}$ of $f^{-1}\left[\varepsilon_{i}-\delta, \varepsilon_{i}+\delta\right]$ onto $f^{-1}\left(\varepsilon_{i}\right)$ such that for $t \in\left[\varepsilon_{i}-\delta, \varepsilon_{i}\right), r_{i}$ restricted to $f^{-1}(t)$ is one-to-one except onto $p_{i}$ and the inverse of $p_{i}$ is a pair of points while if $t \in\left(\varepsilon_{i}, \varepsilon_{i}+\delta\right], r_{i}$ restricted to $f^{-1}(t)$ is one-to-one except onto $p_{i}$ and the inverse of $p_{i}$ is a simple closed curve.

In a similar fashion we define $f$ on $h_{1}\left(B_{1}^{2} \times[2 / 3,1]\right)$ and the holes with ends on $h_{1}\left(B_{1}^{2} \times 2 / 3\right)$.

Definitions. A dendron is a compact locally connected metric continuum which contains no simple closed curve. A point $p$ is of order $\leqq n$ if for each neighborhood $U$ of $p$ there is an open set $V$ with $p \in V \subset U$ such that the boundary (frontier) of $V$ has at most $n$ points. A point is of order $n$ if it is of order $\leqq n$ but not of order $\leqq n-1$. A point of order 1 is an end point of the dendron and a point which is not of order $\leqq 2$ is a branch point. A polyhdral dendron (sometimes called a tree) can have at most a finite number of branch and end points. A dendron with precisely three end points (and hence one branch point) is called a triod.

The following is a variation of Theorem 3.1.

THEOREM 3.2. If $M^{3}$ has precisely three boundary components, there is a piecewise linear monotone map $f$ of $M^{3}$ onto a polyhedral triod $T^{1}$ such that each boundary component of $M^{3}$ is an inverse of an end point of $T^{1}$ and for each $t \in T^{1}, f^{-1}(t)$ is either a 2-manifold or a pinched 2-manifold.

Proof. Let $B_{1}^{2}, B_{2}^{2}$ be two of the boundary components of $M^{3} ; h_{1}$ be a piecewise linear homeomorphism of $B_{1}^{2} \times[0,1]$ onto a cartesian product neighborhood of 
$B_{1}^{2}$ in $M^{3}$ such that for each $x \in B_{1}^{2}, h_{1}\left(B_{1}^{2} \times 0\right)=x$; and $h_{2}$ be a piecewise linear homeomorphism of $B_{2}^{2} \times[0,1]$ onto a cartesian product neighborhood of $B_{2}^{2}$ in $M^{3}$ such that for each $x \in B_{2}^{2}, h_{2}(x \times 0)=x$. We suppose these two cartesian product neighborhoods are disjoint.

Let $H^{3}$ be a hole in $M^{3}-h_{1}\left(B_{1}^{2} \times[0,1)\right)-h_{2}\left(B_{2}^{2} \times[0,1)\right)$ with one end on $h_{1}\left(B_{1}^{2} \times 1\right)$ and the other on $h_{2}\left(B_{2}^{2} \times 1\right)$. It follows by the methods of the proof of Theorem 3.1 that there is a monotone piecewise linear map $f_{1}$ of

$$
H^{3} \cup h_{1}\left(B_{1}^{2} \times[0,1]\right) \cup h_{2}\left(B_{2}^{2} \times[0,1]\right)
$$

into a polyhedral triod $T_{1}^{1}$ such that the inverse of the branch point of $T_{1}^{1}$ is the wedge of two 2-manifolds, the inverse of each other point of $T_{1}^{1}$ is a 2 -manifold. The inverses of the end points of the triod are $h_{1}\left(B_{1}^{2} \times 0\right), h_{2}\left(B_{2}^{2} \times 0\right)$, and the boundary component of $H^{3} \cup h_{1}\left(B_{1}^{2} \times[0,1]\right) \cup h_{1}\left(B_{2}^{2} \times[0,1]\right)$ intersecting Bd $H^{3}$.

It follows from Theorem 3.1 that the piecewise linear map $f_{1}$ of

$$
H^{3} \cup h_{1}\left(B_{1}^{2} \times[0,1]\right) \cup h_{2}\left(B_{2}^{2} \times[0,1]\right)
$$

onto $T_{1}^{1}$ can be extended to a piecewise linear map $f$ of $M^{3}$ onto a larger triod $T^{1}$ satisfying the conditions of Theorem 3.2. The $\operatorname{map} f$ we define is open except on the inverse of the branch point of the triod.

In a certain sense, we proved Theorem 3.2 by combining two boundary components of $M^{3}$. Repetitions of this procedure permits us to prove the following extension of Theorem 3.2.

THEOREM 3.3. If $M^{3}$ has more than one boundary component, there is a piecewise linear monotone map $f$ of $M^{3}$ onto a tree $T^{1}$ such that each point of $T^{1}$ is of order $\leqq 3$, the boundary components of $M^{3}$ are the inverses of end points of $T^{1}$, and for each $t \in T^{1}, f^{-1}(t)$ is either a 2-manifold or a pinched 2-manifold.

Theorem 7.2 of $\$ 7$ gives results about the mapping of $M^{3}$ onto a dendron if $M^{3}$ has no boundary.

4. Decomposition maps onto 2-spheres. In the last section we decomposed $M^{3}$ into 2-manifolds and pinched 2-manifolds by letting these be the point inverses in a map onto a dendron. In $\S 6$ we shall map $M^{3}$ onto a punctured cube. This is done by considering the point inverses in the map onto a dendron and decomposing $M^{3}$ by decomposing each of these inverses. In this section we consider useful decompositions of 2-manifolds and pinched 2-manifolds.

Consider a simplex $\Delta$ which is the join of two simplexes $\Delta_{1}, \Delta_{2}$. Then $\Delta=\Delta_{1} \circ \Delta_{2}$ is the union of segments from $\Delta_{1}$ to $\Delta_{2}$. For each $t \in[0,1]$, let $A_{t}$ be the set of all points such that for some $p_{1} \in \Delta_{1}, p_{2} \in \Delta_{2}, p$ divides the segment from $p_{1}$ to $p_{2}$ in the ratio $t$ to $1-t$. We denote $p$ by $\left(p_{1}, p_{2}, t\right)$ and note that

$$
A_{t}=\left\{\left(p_{1}, p_{2}, t\right) \mid p_{1} \in \Delta_{1}, p_{2} \in \Delta_{2}\right\} \text {. }
$$

Then $A_{0}=\Delta_{1}, A_{1}=\Delta_{2}$, and the midsection $A_{1 / 2}$ is homeomorphic to $\Delta_{1} \times \Delta_{2}$. We use $A_{B}$ to denote $\bigcup_{t \in B} A_{t}$. 
Let $\Delta / \Delta_{1}$ be the cell obtained from $\Delta$ by shrinking $\Delta_{1}$ to a point. To give $\Delta / \Delta_{1}$ a structure we regard it as the union of $A_{[1 / 2,1]}$ and the cone $C$ over $A_{1 / 2}$ from a point $v_{0}$ where $A_{[1 / 2,1]} \cap C=A_{1 / 2}$.

The natural decomposition map $f$ from $\Delta$ to $\Delta / \Delta_{1}$ is the identity on $A_{[1 / 2,1]}$ and for $t \in[0,1 / 2], f\left(p_{1}, p_{2}, t\right)$ is the point that divides the segment from $v_{0}$ to $\left(p_{1}, p_{2}, 1 / 2\right)$ in the ratio $2 t$ to $1-2 t$.

We note that although $\Delta$ is a simplex and $f(\Delta)=\Delta / \Delta_{1}$ is a piecewise linear cell, the $\operatorname{map} f$ is not piecewise linear.

If $L$ is a subcomplex of complex $K, K / L$ results by regarding $L$ as a point. To give $K / L$ a cellular structure, we let $T^{\prime}$ be the first barycentric subdivision of the triangulation of $K$ and note that if $\Delta$ is a simplex in $T^{\prime}$, then either $\Delta$ misses $L$, lies in $L$, or intersects it in a proper face $\Delta_{L}$. The natural decomposition map $f$ is such that $f(L)$ is a vertex $v_{0}$ of $K / L ; f$ is the identity on each simplex of $T^{\prime}$ that misses $L$; for each simplex $\Delta$ of $T^{\prime}$ whose intersection with $L$ is a proper face $\Delta_{L}$ of $\Delta, f$ is the natural decomposition map from $\Delta$ to $\Delta / \Delta_{L}$.

The following result is well known.

THEOREM 4.1. If $M^{2}$ is a compact connected polyhedral 2-manifold, there is a connected polyhedral 1-complex $C^{1}$ in $M^{2}$ such that $M^{2} / C^{1}$ is a polyhedral 2-sphere.

To obtain $C^{1}$, triangulate $M^{2}$ and remove an open 2-simplex Int $\Delta^{2}$ from $M^{2}$. Then $C^{1}$ is obtained by collapsing $M^{2}-$ Int $\Delta^{2}$.

The following two results may be proved in a similar fashion.

THEOREM 4.2. If $\mathrm{N}^{2}$ is a compact connected triangulated pinched 2-manifold with no separating point, there is a connected polyhedral 1-complex $C^{1}$ in the 1skeleton of the triangulation such that $N^{2} / C^{1}$ is a polyhedral 2-sphere and $C^{1}$ is of order 2 at the pinch point.

We note that $C^{1}$ abuts on the pinch point from both sides in $N^{2}$.

THEOREM 4.3. If $\mathrm{N}^{2}$ is a compact connected triangulated pinched 2-manifold which is separated by its pinch point, there is a connected polyhedral 1-complex $C^{1}$ in the 1-skeleton of the triangulation such that $C^{1}$ abuts on the pinch point from both sides and is of order 2 there while $N^{2} / C^{1}$ is the wedge of two polyhedral 2-spheres.

5. Changing pseudo spines. A pseudo spine for a compact connected polyhedral 2-manifold is a connected polyhedron $C$ in $M^{2}$ such that $M^{2} / C$ is topologically a 2-sphere. To get $C$ one might remove an open 2-simplex from $M^{2}$ and collapse the remainder. Note that the pseudo spine differs from a spine for $M^{2}$ in that an open simplex was removed before the collapsing. Also, the collapsing was not necessarily continued as much as possible. For example, a 1-simplex on $S^{2}$ serves as a pseudo spine for it. To emphasize that the pseudo spines we shall be using are 1-dimensional polyhedra, we may call them pseudo 1-spines. 
If $M^{2}$ is a compact, connected, polyhedral 2-manifold, $M^{2} \times[0,1]$ is its cartesian product with an interval, and $C_{0}^{1} \times 0, C_{1}^{1} \times 1$ are pseudo 1-spines of $M^{2} \times 0, M^{2} \times 1$, we shall be interested in finding pseudo 1 -spines for the intermediate layers.

THEOREM 5.1. Suppose $M^{2}$ is a compact connected polyhedral 2-manifold and $C_{0}^{1}, C_{1}^{1}$ are pseudo 1-spines of $M^{2}$. Then $C_{0}^{1}, C_{1}^{1}$ can be extended to a 1-parameter family of pseudo 1-spines $C_{t}^{1}(0 \leqq t \leqq 1)$ of $M^{2}$ such that there is a monotone map $f$ of $M^{2} \times[0,1]$ onto $S^{2} \times[0,1]$ with $f\left(M^{2} \times t\right)$ being equal to $S^{2} \times t$ and $C_{t}^{1} \times t$ being the only nondegenerate inverse in $M^{2} \times t$.

Proof. Let $D_{1}^{2}, D_{2}^{2}, \ldots$ be a sequence of polyhedral disks in $M^{2}$ such that $D_{i}^{2} \subset$ Int $D_{i+1}^{2}$ and $\bigcup D_{i}^{2}=M^{2}-C_{0}^{1}$ and $E_{1}^{2}, E_{2}^{2}, \ldots$ be such a sequence of disks whose union is $M^{2}-C_{1}^{1}$.

Since $E_{1}^{2}$ is a disk which lies on the interior of a larger disk in $M^{2}$ with the larger disk not being a subset of $M^{2}-$ Int $D_{1}^{2}$, there is a piecewise linear isotopy fixed except on this larger disk that pushes $M^{2}-$ Int $D_{1}^{2}$ off $E_{1}^{2}$. Denote this isotopy by $H_{t}(0 \leqq t \leqq 1 / 4)$ where $H_{0}=$ identity and $E_{1}^{2} \cap H_{1 / 4}\left(M^{2}-\right.$ Int $\left.D_{1}^{2}\right)=0$. Then $H_{1 / 4}\left(D_{1}^{2}\right)$ - Int $E_{1}^{2}$ is an annulus and $H_{t}(0 \leqq t \leqq 1 / 4)$ can be extended to a piecewise linear isotopy $H_{t}(0 \leqq t \leqq 1 / 2)$ such that $H_{1 / 2}\left(D_{1}^{2}\right)=E_{1}^{2}$.

The isotopy $H_{t}(0 \leqq t \leqq 1 / 2)$ can be extended to a piecewise linear isotopy $H_{t}$ $(0 \leqq t \leqq 3 / 4)$ so that $H_{3 / 4}\left(D_{2}^{2}\right)=E_{2}^{2}$ and for $x \in D_{1}^{2}$ and $1 / 2 \leqq s \leqq 3 / 4, H_{s}(x)=H_{1 / 2}(x)$.

Similarly, we extend $H_{t}$ to $H_{t}(0 \leqq t<1)$. In general, $H_{t}$ is such that $H_{t_{i}}\left(D_{i}\right)=E$ for $t_{i}=\left(1-1 / 2^{i}\right)$ and $H_{s}=H_{t_{i}}$ on $D_{i}$ for $s \geqq\left(1-1 / 2^{i}\right)$.

For $0 \leqq t<1, C_{t}^{1}=H_{t}\left(C_{0}^{1}\right)$.

Let $f_{0}$ be a decomposition map of $M^{2}$ onto $S^{2}$ such that $C_{0}^{1}$ is the only nondegenerate inverse. The map $f$ of $M^{2} \times[0,1)$ is defined by $f(x \times t)=f_{0} H_{t}^{-1}(x) \times t$. It is extended to $M^{2} \times 1$ by continuity.

Questions. It would appear that Theorem 5.1 is a special case of some more general theorem. If $f$ is a map of a compact continuum $X$ onto $[0,1]$ such that the point inverses are all homeomorphic to each other, under what condition can it be concluded that $X$ is homeomorphic to $f^{-1}(0) \times[0,1]$ ? If $g$ is a monotone map of $Y \times[0,1]$ onto $Z$ such that the $g(Y \times t)$ 's are homeomorphic but no two intersect, under what condition is $Z$ homeomorphic to $g(Y \times 0) \times[0,1]$ ? Is it enough that each $f(Y \times t)$ be a 2 -sphere? What if each $Y \times t$ contain at most one nondegenerate point inverse? Something needs to be imposed because if $P$ is a pseudo arc, $P^{\prime}$ is a nondegenerate proper subcontinuum of $P \times 0$, then $(P \times[0,1]) / P^{\prime}$ is not homeomorphic to $P \times[0,1]$ since it contains a triod. However it is known [2] that $(P \times 0) / P^{\prime}$ is homeomorphic. to $P$.

It is to be noted that the map $f$ is not piecewise linear and is especially bad at $t=1$. Can we find such an $f$ that has the nice properties of a natural decomposition map?

6. Decomposing 3-manifolds with boundaries. Suppose $B_{0}^{3}, B_{1}^{3}, \ldots, B_{n}^{3}$ are 
polyhedral 3-cells such that $B_{i}^{3} \subset$ Int $B_{0}^{3}$ for $i=1,2, \ldots, n$ and $B_{i}^{3} \cap B_{j}^{3}=0$ for $0<i<j \leqq n$. Then $B_{0}^{3}-\bigcup_{i=1}^{n}$ Int $B_{i}^{3}$ is a polyhedral punctured cube.

If the boundary components of $M^{3}$ are already nicely decomposed with the decomposition space of each being a 2-sphere, we shall be interested in extending the decomposition to $M^{3}$ so that the decomposition space is a punctured cube.

THEOREM 6.1. Suppose $M^{3}$ has precisely two boundary components $B_{0}^{2}$, $B_{1}^{2}$ with pseudo 1-spines $C_{0}^{1}, C_{1}^{1}$. Then there is a monotone map $g$ of $M^{3}$ onto $S^{2} \times[0,1]$ such that $g^{-1}\left(S^{2} \times 0\right)=B_{0}^{2}, g^{-1}\left(S^{2} \times 1\right)=B_{1}^{2}$, each $g^{-1}\left(S^{2} \times t\right)$ is either a polyhedral 2-manifold or polyhedral pinched 2-manifold, $C_{0}^{1}$ and $C_{1}^{1}$ are point inverses, each nondegenerate point inverse is a polyhedral 1-complex, and the image of the union of the nondegenerate point inverses is an arc from $S^{2} \times 0$ to $S^{2} \times 1$ that intersects each $S^{2} \times t$ only once.

Proof. Let $f$ be a monotone piecewise linear map of $M^{3}$ onto $[0,1]$ as described in Theorem 3.1 such that $f^{-1}(0)=B_{0}^{2}, f^{-1}(1)=B_{1}^{2}$, and each inverse is either a 2-manifold or a pinched 2-manifold. We plan to define $g$ so that $g f^{-1}(t)=S^{2} \times t$. To get such a $g$ we need to get a 1-complex $C_{t}^{1}$ on each $f^{-1}(t)$ so that $f^{-1}(t) / C_{t}^{1}$ is homeomorphic to $S^{2}$. We shall first describe the $C_{t}^{1}$ 's on the pinched inverses, then on the inverses near these pinched inverses, and finally use Theorem 5.1 to fill in the gaps.

Since $f$ is piecewise linear, there are only a finite number of pinched 2-manifolds among the inverses. Let $0=t_{1}<t_{2}<\cdots<t_{3 n+2}=1$ be such that the pinched inverses are the $f^{-1}\left(t_{3 i}\right)$ 's $(i=1,2, \ldots, n)$. For each $i=1,2, \ldots, n$, let $C_{t_{3 i}}^{1}$ be a polyhedral 1-complex in $f^{-1}\left(t_{3 i}\right)$ such as guaranteed by Theorem 4.2 so that $f^{-1}\left(t_{3 i}\right) / C_{t_{3 i}}^{1}$ is a 2 -sphere.

In the proof of Theorem 3.1 we analyzed the nature of the map $f$ in the neighborhood of the pinched inverses. There is a projection of the $f^{-1}(t)$ 's near $f^{-1}\left(t_{3 i}\right)$ onto $f^{-1}\left(t_{3 i}\right)$ so that the projection is one-to-one except onto the pinched point and the inverse of the pinched point under the projection is either a pair of points or a polyhedral simple closed curve. For $t \in\left[t_{3 i-1}, t_{3 i+1}\right]$ let $C_{t}^{1}$ be the inverse of $C_{t_{3 i}}^{1}$ under the projection of $f^{-1}(t)$ onto $f^{-1}\left(t_{3 i}\right)$. There is a map $g_{i}$ of $f^{-1}\left[t_{3 i-1}, t_{3 i+1}\right]$ onto $S^{2} \times\left[t_{3 i-1}, t_{3 i+1}\right]$ such that $g f^{-1}(t)=S^{2} \times t$ and $C_{t}^{1}$ is the only nondegenerate inverse in $g^{-1}\left(S^{2} \times t\right)$.

Although there is a piecewise linear homeomorphism of $f^{-1}\left[t_{3 j-2}, t_{3 j-1}\right]$ for $j=1,2, \ldots, n+1$ onto $f^{-1}\left(t_{3 j-2}\right) \times\left[t_{3 j-2}, t_{3 j-1}\right]$, there is no assurance that $C_{t_{3 j-2}}^{1}$ and $C_{t_{3 j-1}}^{1}$ line up. However Theorem 5.1 shows that polyhedral $C_{t}^{1}$ 's can be selected for $t \in\left(t_{3 j-2}, t_{3 j-1}\right)$ so as to insure that there is a level preserving monotone map of $f^{-1}\left[t_{3 j-2}, t_{3 j-1}\right]$ onto $S^{2} \times\left[t_{3 j-2}, t_{3 j-1}\right]$ so that the only nondegenerate inverses are the $C_{t}^{1}$ 's.

The map $g$ is obtained by patching together the various maps of the $f^{-1}\left[t_{3 i-1}, t_{3 i+1}\right]$ onto the $S^{2} \times\left[t_{3 i-1}, t_{3 i+1}\right]$ for $i=1,2, \ldots, n$ and the maps of the $f^{-1}\left[t_{3 j-2}, t_{3 j-1}\right]$ onto the $S^{2} \times\left[t_{3 j-2}, t_{3 j-1}\right]$ for $j=1,2, \ldots, n+1$. 
THEOREM 6.2. If $M^{3}$ has only one boundary component $B^{2}$ and $C^{1}$ is a pseudo 1-spine of $B^{2}$, then there is a monotone map $g$ of $M^{3}$ onto a polyhedral 3-cell $B^{3}$ such that $g^{-1}\left(\mathrm{Bd} B^{3}\right)=B^{2}$, each nondegenerate inverse is a polyhedral 1-complex, $C^{1}$ is the only such inverse on $B^{2}$, and the image under $g$ of the union of the nondegenerate inverses is a polyhedral arc in $B^{3}$.

Proof. A polyhedral open ball is removed from $M^{3}$ to change it to a 3-manifold with two boundary components. A polygonal arc is selected to serve as a pseudo 1-spine for the new boundary component. Theorem 6.1 assures us that there is a suitable map of the remainder onto $S^{2} \times[0,1]$. This map may be extended to all of $M^{3}$ to get the required map onto $B^{3}$.

THEOREM 6.3. Suppose $M^{3}$ has boundary components $B_{1}^{2}, B_{2}^{2}, \ldots, B_{n}^{2}(n \geqq 2)$ with polyhedral pseudo 1-spines $C_{1}^{1}, C_{2}^{1}, \ldots, C_{n}^{1}$. Then there is a monotone map $g$ of $M^{3}$ onto a polyhedral punctured cube $K^{3}$ with boundary components $E_{1}^{2}, E_{2}^{2}, \ldots$, $E_{n}^{2}$ such that $g^{-1}\left(E_{i}^{2}\right)=B_{i}^{2}, C_{i}^{1}$ is the only nondegenerate inverse on $B_{i}^{2}$, each nondegenerate inverse is a polyhedral 1-complex, and the image under $g$ of the union of the nondegenerate inverses is a tree in $K^{3}$ each of whose ends is on $\mathrm{Bd} K^{3}$.

Proof. Let $f$ be a piecewise linear map such as guaranteed by Theorem 3.3 and described in the proofs of Theorems 3.1 and 3.2 such that $f\left(M^{3}\right)$ is a tree $T^{1}$, each branch point of $T^{1}$ is of order 3 , the inverses of the end points of $T^{1}$ are the boundary components of $M^{3}$, and for each $x \in T^{1}, f^{-1}(x)$ is either a 2-manifold or a pinched 2-manifold.

Let $f^{-1}\left(x_{1}\right), f^{-1}\left(x_{2}\right), \ldots, f^{-1}\left(x_{n-2}\right)$ be the pinched 2-manifolds among the inverses which are separated by their pinch points. Each $f^{-1}\left(x_{i}\right)$ separates $M^{3}$ into three parts. Assign a 1-complex $C_{x_{i}}^{1}$ to $f^{-1}\left(x_{i}\right)$ which satisfies the conditions of Theorem 4.3.

Let $T_{1}^{1}, T_{2}^{1}, \ldots, T_{n-2}^{1}$ be mutually exclusive trees in $T^{1}$ about the $x_{i}$ 's such that the branch point of $T_{i}^{1}$ is the only point of it that has an inverse which is pinched and no end point of $T^{1}$ is in any $T_{i}^{1}$.

For each $x \in T_{i}^{1}$, there is a natural projection of $f^{-1}(x)$ into $f^{-1}\left(x_{i}\right)$. From two sides, the projection is one-to-one onto manifolds in $f^{-1}\left(x_{i}\right)$ and from the third side the projection is one-to-one onto $f^{-1}\left(x_{i}\right)$ except onto the pinch point and the inverse of the pinch point is a polyhedral simple closed curve.

For the $f^{-1}(x)$ 's that project one-to-one onto a 2-manifold in $f^{-1}\left(x_{i}\right)$, we let $C_{x}^{1}$ be the inverse under the projection of the part of $C_{x_{i}}^{1}$ in this 2-manifold.

For the $f^{-1}(x)$ 's that project onto all of $f^{-1}\left(x_{i}\right)$, we let $C_{x}^{1}$ be the inverse of $C_{x_{i}}^{1}$ under the projection with an open arc removed from the inverse of the pinch point under the projection so that $f^{-1}(x) / C_{x}^{1}$ is a 2-sphere rather than the wedge of two 2-spheres. There is a monotone map $g_{1}$ of $f^{-1}\left(T_{i}^{1}\right)$ onto a polyhedral punctured cube $K_{i}^{3}$ with three boundary components such that $C_{x}^{1}$ is the only nondegenerate inverse intersecting $f^{-1}(x)$. 
Consider the components of the part of $T^{1}$ for which $C^{1}$ 's have not been assigned to the inverses of its points. There are only a finite number of such components and the closure of each is an arc $A$. Already $C^{1}$ 's have been assigned to the inverses of the ends of $A$. Theorem 6.1 provides $C^{1}$ 's to the inverses of other points of $A$ as well as a monotone map of $f^{-1}(A)$ into a hollow ball. The map $g$ promised by Theorem 6.3 follows by patching together the monotone maps $g_{i}$ on the $f^{-1}\left(T_{i}^{1}\right)$ 's and those on the $A$ 's.

7. Extending monotone mappings. In this section we suppose $M^{3}$ is without boundary and $X$ is a closed proper subset of $M^{3}$ such that if $U$ is a connected open subset of $M^{3}$, then either $U-(X \cap U)$ is connected or $X$ intersects the boundary (frontier) of $U$.

The principal result (Theorem 7.3) of this section is that there is a monotone map of $M^{3}$ onto $S^{3}$ that has the components of $X$ as point inverses. Before proving this result, we prove some theorems about $X$.

THEOREM 7.1. There is a decreasing sequence $M_{1}^{3}, M_{2}^{3}, \ldots$ such that $X=M_{1}^{3}$ $\cap M_{2}^{3} \cap \cdots$ where each $M_{i}^{3}$ is a 3-manifold with boundary which is a polyhedron in $M^{3}, M_{i+1}^{3} \subset M_{i}^{3}$, and each component of $M_{i}^{3}$ has a connected boundary.

Proof. Suppose $M_{i}^{3}$ has been constructed. We prove the theorem by showing how to get an $M_{i+1}^{3}$ in any open subset $V$ of Int $M_{i}^{3}$ which contains $X$.

Let $T$ be a triangulation of $M^{3}$ of mesh less than one-half the distance from $X$ to $M^{3}-V$ and $T^{\prime \prime}$ be the second barycentric subdivision of $T$. Let $N^{3}$ be the union of the closed simplexes of $T$ which intersect $X$ and $N_{1}^{3}$ be the union of the closed simplexes of $T^{\prime \prime}$ which intersect $N^{3}$. Then $N_{1}^{3}$ is a regular neighborhood of $N^{3}$ and is hence a polyhedral 3-manifold with boundary ([7], [10], [12]). If $C^{3}$ is a component of $N_{1}^{3}$, it may have several boundary components, but boring holes in $C^{3}$ which miss $X$ reduces the number of boundary components to one. Hence $M_{i+1}^{3}$ is obtained from $N_{1}^{3}$ by boring holes.

The following result follows from Theorem 7.1 and repeated applications of Theorem 3.3.

THEOREM 7.2. There is a dendron $D^{1}$ and a monotone map $f$ of $M^{3 \cdot}$ onto $D^{1}$ such that each point of $D^{1}$ is of order $\leqq 3, D^{1}$ is locally polyhedral mod its set of end points, the components of $X$ are the inverses of the endpoints of $D^{1}$, the inverse of each non end point is either a polyhedral 2-manifold or a polyhedral pinched 2-manifold, and $f$ is locally piecewise linear mod $X$.

THEOREM 7.3. There is a monotone map $g$ of $M^{3}$ onto $S^{3}$ such that each component of $X$ is a point inverse, $g(X)$ lies on a straight segment, and each nondegenerate point inverse outside $X$ is a polyhedral 1-complex. In fact, there are also a dendron $D^{1}$ in $S^{3}$ and a monotone map of $M^{3}$ onto $D^{1}$ such that $D^{1}$ is locally polyhedral mod its set of end points, each branch point of $D^{1}$ is of order $3, X$ is the inverse under $f$ of the set of end points of $D^{1}, f=g$ on $X$ and on each nondegenerate point inverse under $g$, 
and if $x$ is a non end point of $D^{1}, f^{-1}(x)$ is either a polyhedral 2-manifold or pinched 2-manifold, $f^{-1}(x)$ contains at most one nondegenerate point inverse under $g$, and $g f^{-1}(x)$ is either a 2-sphere or the wedge of two 2-spheres.

Proof. The dendron $D^{1}$ and the map $f$ are as given in Theorem 7.2, (except that the embedding of $D^{1}$ in $S^{3}$ is yet to be defined).

Let $T_{1}^{1}, T_{2}^{1}, \ldots$ be a monotone increasing sequence of trees in $D^{1}$ such that each point of order $\leqq 2$ of $T_{i}^{1}$ is a point of order 2 of $T_{i+1}^{1}$, each inverse under $f$ of an end point of $T_{i}^{1}$ is a 2-manifold, and $\bigcup T_{i}^{1}$ contains each non end point of $D^{1}$.

By Theorem 6.3 there is a monotone map $g_{1}$ of $f^{-1}\left(T_{1}^{1}\right)$ onto a punctured cube $K_{1}^{3}$ such that each nondegenerate point inverse under $g_{1}$ is a polyhedral 1-complex, the image of the set of nondegenerate point inverses is a tree $T_{1^{\prime}}^{1}$, and for each $x \in T_{1}^{1}, f^{-1}(x)$ contains exactly one such 1-complex, and $g_{1} f^{-1}(x)$ is either a 2 sphere or the wedge of 2-spheres.

Let $A$ be a straight segment in $S^{3}$. We suppose that $K^{3}$ lies in $S^{3}$, each component of $S^{3}-K^{3}$ is of diameter less than $\frac{1}{2}$ and intersects $A, T_{1^{\prime}}^{1}=T_{1}^{1}, f=g$ on $f^{-1}\left(T_{1}^{1}\right)$.

Let $T_{2, i}^{1}(i=1,2, \ldots, m)$ be the closures of the components of $T_{2}^{1}-T_{1}^{1}$. It follows from Theorem 6.3 that there is a monotone map $g_{2, i}$ of $f^{-1}\left(T_{2, i}^{1}\right)$ onto a punctured cube $K_{2, i}^{3}$ such that each nondegenerate point inverse under $g_{2, i}$ is a polyhedral 1-complex, the image of the union of these 1-complexes is a tree $T_{2, i^{\prime}}^{1}$, and for each $x \in T_{2, i}^{1}, f^{-1}(x)$ contains exactly one such 1-complex, while $g_{2, i} f^{-1}(x)$ is either a 2-sphere or the wedge of two 2-spheres.

We suppose that $K_{2, i}^{3}$ lies in $S^{3}$ so that only one component of $S^{3}-K_{2, i}^{3}$ is of diameter more than $1 / 4$, each component of $S^{3}-K_{2, i}^{3}$ intersects $A$, and for $x_{i}=T_{1}^{1} \cap T_{2, i}^{1}, g_{1}, g_{2, i}$ agree on $f^{-1}\left(x_{i}\right)$ with $g_{1} f^{-1}\left(x_{i}\right)=g_{2, i} f^{-1}\left(x_{i}\right)=K_{1}^{3} \cap K_{2, i}^{3}$, $T_{2, i^{\prime}}^{1}=T_{2, i}^{1}$, and $f=g$ on $f^{-1}\left(T_{2}^{1}\right)$.

For simplicity we use $g$ to designate $g_{1}$ and the $g_{2, i}$ 's. The map $g$ is extended to $T_{3}^{1}, T_{4}^{1}, \ldots$ so that each component of $S^{3}-g f^{-1}\left(T_{i}^{1}\right)$ is of mesh less than $1 / 2^{i}$ and intersects $A$. We place $D^{1}$ so that $f=g$ on $f^{-1}\left(D^{1}\right)$. Then $g$ is extended to $M^{3}$ so that if $y_{0}$ is an end point of $D^{1}$ and $y_{i}$ is the end point of $T_{i}^{1}$ toward $y_{0}, g f^{-1}\left(y_{0}\right)$ is the intersection of the small balls in $S^{3}$ bounded by the $g f^{-1}\left(y_{i}\right)$ 's.

The unicoherence of $S^{3}$ permits us to weaken the restrictions on $X$ (where such a weakening would not be possible for such a 3-manifold as $S^{2} \times S^{1}$ ). Hence, by ignoring details about $f$ we may state Theorem 7.3 for $S^{3}$ as follows.

THEOREM 7.4. If $Y$ is a closed subset of $S^{3}$ no component of which separates $S^{3}$, there is a monotone map of $S^{3}$ onto itself such that the components of $Y$ are point inverses, each nondegenerate point inverse not in $Y$ is a polyhedral 1-complex, and the image of the union of $Y$ and these nondegenerate point inverses is a dendron.

We can deal with noncompact 3-manifolds since each connected noncompact 3-manifold is the union of a monotone increasing sequence of connected compact 
3-manifolds with boundaries. For example, for Euclidean 3-space $E^{3}$ we have the following result.

THEOREM 7.5. Suppose $Y$ is a closed subset of $E^{3}$ such that each component of $Y$ is compact but does not separate $E^{3}$. Then there is a compact monotone map of $E^{3}$ onto itself such that the components of $Y$ are point inverses, and nondegenerate point inverses not in $Y$ are polyhedral 1-complexes. The image of the union of $Y$ and the nondegenerate point inverses is a closed set whose 1-point compactification is a dendron with a closed set of end points.

QUESTIONS. In [11] Whyburn raises the question as to whether or not each monotone map of $E^{3}$ onto itself need be compact. Could it be shown to be compact in case each point inverse is either a 1-complex or a component of $Y$ ? Would it help to know that the image of the union of the nondegenerate point inverses lies in a closed set whose 1-point compactification is a dendron?

It is to be noted that the maps $g$ of Theorems $7.4,7.5$ were not necessarily cellular since some of the 1-complexes which were point inverses might contain cycles. Could one obtain a cellular $g$ if the components of $Y$ were pointlike? In particular, what happens if $Y$ is the union of the tame arcs in the dogbone decomposition?

Definitions. Euclidean $n$-space $(n>1)$ has only one end. An end of a connected, locally compact, noncompact metric space $S$ may be defined as an equivalence class of sequences $U_{1}, U_{2}, \ldots$ where $U_{i}$ is a nonnull connected open subset of $X$ with a compact boundary (frontier),

$$
\overline{U_{i+1}} \subset U_{i} \text {, and } \bigcap U_{i}=0 .
$$

The sequence $U_{1}, U_{2}, \ldots$ is in the same equivalence class as $V_{1}, V_{2}, \ldots$ if and only if the $V_{i}$ 's satisfy similar conditions, each $U_{i}$ contains a $V_{j}$ and each $V_{i}$ contains a $U_{j}$. Hence, $S$ has one end if for each compact set $C$, the closure of $S-C$ has precisely one noncompact component.

For other noncompact 3-manifolds, our methods give the following result.

THEOREM 7.6. Suppose $W^{3}$ is a noncompact, connected, polyhedral, 3-manifold without boundary and $Y$ is a closed subset of $W^{3}$ such that each component of $Y$ is compact and if $U$ is a connected open subset of $W^{3}$, either $Y$ intersects the boundary of $U$ or $U-(Y \cap U)$ is connected. Then there is a straight segment in $S^{3}$, a dendron $D^{1}$ which is locally polyhedral mod its set of end points and which intersects $A$ in its set of end points, a closed subset $C^{0}$ of the set of end points of $D^{1}$, and a monotone map $g$ of $W^{3}$ onto $S^{3}-C^{0}$ such that the components of $Y$ are point inverses of the end points of $D^{1}$ not in $C^{0}$, the nondegenerate point inverses not in $Y$ are polyhedral 1-complexes in $W^{3}$, and the image of the union of $Y$ and the nondegenerate point inverses is $D^{1}-C^{0}$.

The points $C^{0}$ correspond to the ends of $W^{3}$. 
8. Monotone maps that cannot be extended. It would be interesting to know which monotone decompositions of a portion of a 3-manifold can be extended to the whole 3-manifold so that the decomposition space is a 3-manifold.

Suppose $C_{1}, C_{2}$ are two linking round circles in a simplex of $S^{3}$ and $G$ is a decomposition of $C_{1} \cup C_{2}$ whose only nondegenerate element is $C_{2}$. Is there a monotone upper semicontinuous decomposition of $S^{3}$ onto itself such that each element of $G$ is an element of this decomposition? Is there a monotone map $g$ of $S^{3}$ onto itself that is a homeomorphism on $C_{1}$ and sends $C_{2}$ to a point off $g\left(C_{1}\right)$ ? The example on p. 7 of [5] gives an affirmative answer to the second question while the following theorem gives a negative answer to the first.

The following theorem is included for simplicity. It is a special case of the more complicated Theorem 8.4.

THEOREM 8.1. There is no monotone upper semicontinuous decomposition of $S^{3}$ onto itself such that the elements of $G$ are elements of this decomposition.

Proof. Assume the decomposition can be extended. Then there is a monotone map $g$ of $S^{3}$ onto itself such that the elements of $G$ are point inverses under $g$.

Let $J$ be a simple closed curve in $S^{3}-g\left(C_{1}\right)$ that cannot be shrunk to a point in $S^{3}-g\left(C_{1}\right)$. We show that there is no map $g$ by showing that $J$ can be shrunk to a point in $S^{3}-g\left(C_{1}\right)$.

It follows from the monotonicity of $g$ and the local Euclidean structure of $S^{3}$ that for each open set $U$ containing $J$ there is a homeomorphism $h$ of $J$ into $g^{-1}(U)$ such that the identity on $J$ is homotopic to $g h$ in $U$. We suppose that $h$ is selected for an open subset $U$ containing $J$ such that $U \cap g\left(C_{1}\right)=0$.

Let $H_{t}(0 \leqq t \leqq 1)$ be an isotopy of $S^{3}-C_{1}$ into $S^{3}-C_{1}$ such that $H_{0}=$ Identity and $H_{1}\left(S^{3}-C_{1}\right)=C_{2}$. Then a homotopy shrinking $J$ to a point in $S^{3}-g\left(C_{1}\right)$ spends the first part of its parameter pulling the identity on $J$ to $g h$ and the last part as $g H_{t} h(0 \leqq t \leqq 1)$.

EXAMPLE. One cannot weaken Theorem 8.1 by replacing “onto itself" by "onto a subset of itself". If one considers $S^{3}$ as the join of circles $C_{1}$ and $C_{2}$, one sees that there is a monotone decomposition of $S^{3}$ onto a disk that homeomorphically sends $C_{1}$ to the boundary of the disk and $C_{2}$ to the center. Other point inverses are simple curves that link $C_{1}$.

Definitions. Suppose $U$ is an open subset of a polyhedral 3-manifold and $J^{1}$ is polygonal simple closed curve in $U$. We say that $n J^{1}$ bounds (mod the integers) in $U$ if $J$ can be regarded as an oriented 1-cycle so that there is some oriented complex $C^{2}$ in $U$ such that $\partial C^{2}=n J^{1}$. By adjusting $C^{2}$ to remove certain multiple points we may suppose with no loss of generality that it is locally a 2-manifold off $J^{1}$ and that at $J^{1}$ it locally resembles $n$ sheets with a common edge.

Notice that in considering complexes, we are using the geometric approach rather than an abstract one and regard a simplex as a geometric object (perhaps oriented), a complex as a geometric object (with perhaps its simplexes oriented), and the 
boundary of an oriented complex as an oriented complex of one lower dimension.

If $M^{2}$ is a connected polyhedral oriented 2-manifold in an oriented polyhedral 3-manifold, $M^{2}$ locally has two sides. If $J^{1}$ is a polyhedral simple closed curve that intersects $M^{2}$ in only a finite number of points, it pierces it at each of these, and pierces it $n$ more times in one direction than in the other, we call $n$ the (unsigned) piercing number of $J^{1}$ and $M^{2}$. Similarly, if $J^{1}$ intersects a 2-complex $C^{2}$ in only a finite number of points, none of these are on the 1-skeleton of $C^{2}$, and it pierces $C^{2}$ at each of these points, we may define the piercing number of $J^{1}$ and $C^{2}$ in a similar fashion.

THEOREM 8.2. Suppose $J$ is a simple closed curve (perhaps wild) in a polyhedral 3-manifold $W^{3}$ such that $J$ can be shrunk to a point in $W^{3}$. Then for each point $p \in J$ and each neighborhood $U$ of $p$ there is polyhedral simple closed curve $K^{1} \subset U-J$ such that no positive multiple of $K^{1}$ bounds in $W^{3}-J$.

Proof. Let $J$ be the union of two arcs $p x_{0} q, p x_{1} q$ and $B_{1}^{3}, B_{2}^{3}$ be polyhedral 3-cells in $W^{3}$ such that $p \in \operatorname{Int} B_{2}^{3} \subset B_{3}^{3} \subset\left(\operatorname{Int} B_{1}^{3} \cap U\right), q \in W^{3}-B_{2}^{3}$, and there is a homotopy pulling $J$ into $B_{1}^{3}$ that is the identity on $J \cap B_{2}^{3}$.

The 2-sphere $\mathrm{Bd} B_{2}^{3}$ is the union of two polyhedral disks $B_{0}^{2}, B_{1}^{2}$ such that $B_{0}^{2}$ $\cap B_{1}^{2}=\mathrm{Bd} B_{0}^{2}=\mathrm{Bd} B_{1}^{2}, p x_{0} q \cap \mathrm{Bd} B_{2}^{3} \subset \mathrm{Int} B_{0}^{2}$, and $p x_{1} q \cap \mathrm{Bd} B_{2}^{3} \subset$ Int $B_{1}^{2}$. Then $K^{1}=\mathrm{Bd} B_{0}^{2}=\mathrm{Bd} B_{1}^{2}$ is the required polyhedral simple closed curve.

Suppose $n$ is positive integer such that $n K^{1}$ bounds in $W^{3}-J$. There is a polyhedral simple closed curve $J_{1}^{1}$ close to $J$ so that $n K^{1}$ bounds in $W^{3}-J_{1}^{1}, J_{1}^{1}$ is the union of arcs $p y_{0} q, p y_{1} q$ such that $p y_{0} q \cap \mathrm{Bd} B_{2}^{3} \subset \operatorname{Int} B_{0}^{2}, p y_{1} q \cap \operatorname{Bd} B_{2}^{3} \subset \operatorname{Int} B_{1}^{2}$, and there is a homotopy pulling $J_{1}^{1}$ into $B_{1}^{3}$ that is the identity on $J_{1}^{1} \cap B_{2}^{3}$.

We suppose that the homotopy $H_{t}(0 \leqq t \leqq 1)$ pulling $J_{1}^{1}$ into $B_{1}^{3}$ is such that there is a finite sequence $0=t_{0}<t_{1}<t_{2}<\cdots<t_{k}=1$ such that each $H_{t_{\mathrm{i}}}$ is a homeomorphism, $H_{0}=$ Identity, $H_{1}\left(J_{1}^{1}\right) \subset \operatorname{Int} B_{1}^{3}$, and $H_{t_{i}}=H_{t_{i+1}}$ except on an arc $A_{i}^{1}$ of $J_{1}^{1}$ such that $H_{t_{i}}\left(A_{i}^{1}\right) \cup H_{t_{i+1}}\left(A_{i}^{1}\right)$ bounds a polyhedral disk $D_{i}^{2}$ in $W^{3}-B_{2}^{3}$. Changing a 2-complex in $W^{3}-J_{1}^{1}$ bounded by $n K^{1}$ by adding oriented handles near the polyhedral disks $D_{i}^{2}$ produces in each $W^{3}-H_{t_{i}}\left(J_{1}^{1}\right)$ a 2-complex bounded by $n K^{1}$. In particular, $n K^{1}$ bounds in $W^{3}-H_{1}\left(J_{1}^{1}\right)$.

As a result of the homotopy discussed in the last paragraph, we suppose with no loss of generality that $J_{1}^{1} \subset \operatorname{Int} B_{1}^{3}$. Let $C^{2}$ be an oriented 2-complex in $W^{3}-J_{1}^{1}$ such that $\partial C^{2}=n K^{1}$. Suppose $B_{1}^{2}$ is oriented so that $C^{2}+n B_{1}^{2}$ is a 2-cycle. (We regard $n B_{1}^{2}$ as $n$ oriented disks all nearly parallel and with the same boundary.) Since $J_{1}^{1}$ misses $C^{2}$ and when ordered $p y_{1} q y_{0} p$ pierces $B_{1}^{2}$ once more in going into $B_{2}^{3}$ than in going out, the piercing number of $J_{1}^{1}$ and $C^{2} \cup n B_{1}^{2}$ is $n$, where in lieu of an orientation of $W^{3}$ we use an orientation of $B_{1}^{3}$. Let $M_{1}^{2}$ be an oriented polyhedral 2-manifold obtained from $C^{2}+n B_{1}^{2}$ so that $J_{1}$ pierces $M_{1}^{2} n$ more times in one direction than it does in the other. We can talk of the direction of the piercing since $n B_{1}^{2} \subset B_{1}^{3}$.

Let $M_{2}^{2}$ be a polyhedral orientable 2-manifold in Int $B_{1}^{3}$ such that $\mathrm{Bd} M_{2}^{2}=J_{1}^{1}$. 
We suppose that $M_{1}^{2}, M_{2}^{2}$ are in such general positions that their intersection is a 1-manifold with boundary along which $M_{1}^{2}$ and $M_{2}^{2}$ locally cross.

Let $A_{1}^{1}, A_{2}^{1}, \ldots, A_{k}^{1}$ be the components of $M_{1}^{2} \cap M_{2}^{2}$ that are arcs. Remove a tubular neighborhood of $A_{1}^{1}$ from $M_{2}^{2}$ so as to change $M_{2}^{2}$ to an oriented polyhedral 2-manifold with boundary $M_{3}^{2}$. There is no assurance that $M_{3}^{2}$ is connected but we suppose it retains the orientation of $M_{2}^{2}$. Certainly, $\mathrm{Bd} M_{3}^{2}$ is not connected. The removal reduces by two the number of points in $M_{1}^{2} \cap \mathrm{Bd} M_{2}^{2}$. However, since the piercing was from different sides, this leaves the piercing number of $\mathrm{Bd} M_{3}^{2}$ and $M_{1}^{2}$ equal to $n$. By removing tubular neighborhoods about the others $A_{i}^{1}$ 's, we arrive at the contradiction that there is a 2-manifold with boundary $M_{k+2}^{2}$ such that $\mathrm{Bd} M_{k+2}^{2}$ misses $M_{1}^{2}$ but its piercing number with $M_{1}^{2}$ is $n$. Hence, our assumption that $n K^{1}$ bounds in $W^{3}-J$ is false.

Note that in proving Theorem 8.2 we made two uses of the fact that $J$ can be shrunk to a point in $W^{3}$-first, that $J$ can be pulled into the orientable part $B_{1}^{3}$ of $W^{3}$ and second, that $J$ bounds. If $W^{3}$ is already orientable we do not need to pull $J$ into $B_{1}^{3}$ and hence obtain the following variation of Theorem 8.2.

THEOREM 8.3. If $J$ is a simple closed curve in a polyhedral, orientable 3-manifold $W^{3}$ and $J$ lies in an open subset $U$ of $W^{3}$, there is a polyhedral simple closed curve $K^{1}$ in $U-J$ such that no positive multiple of $K^{1}$ bounds in $W^{3}-J$.

Proof. As in the proof of Theorem 8.2 we express $J$ as the union of two arcs $p x_{0} q, p x_{1} q$ and let $B^{3}$ be polyhedral 3-cell such that $p \in \operatorname{Int} B^{3} \subset B^{3} \subset U$ and $q \in W^{3}-B^{3}$. We express $\mathrm{Bd} B^{3}$ as the union of two polyhedral disks $B_{0}^{2}, B_{1}^{2}$ such that $B_{0}^{2} \cap B_{1}^{2}=\mathrm{Bd} B_{0}^{2}=\mathrm{Bd} B_{1}^{2}, p x_{0} q \cap \mathrm{Bd} B^{3} \subset$ Int $B_{0}^{2}$, and $p x_{1} q \cap \mathrm{Bd} B_{2}^{3} \subset$ Int $B_{1}^{2}$. Then $\mathrm{Bd} B_{0}^{2}=\mathrm{Bd} B_{1}^{2}=K_{1}^{1}$ is a candidate for $K^{1}$.

If $n$ is a positive integer such that $n K_{1}^{1}$ bounds in $W^{3}-J$, we let $C^{2}$ be a 2complex in $W^{3}-J$ such that $\partial C^{2}=n K_{1}^{1}$. Suppose $B_{1}^{2}$ is oriented so that $C^{2}+n B_{1}^{2}$ is a 2-cycle.

Let $K_{2}^{1}$ be a polygonal simple closed curve in $U-J$ so oriented and so close to $J$ that $K_{2}^{1}$ misses $C^{2}, K_{2}^{1}$ intersects $B_{1}^{2}$ in only a finite number of places, pierces it at each of these, and it pierces $B_{1}^{2}$ once more in going into $B^{3}$ than in going out. Let $M_{1}^{2}$ be an oriented polyhedral 2-manifold obtained from $C^{2}+n B_{1}^{2}$ so that $K_{2}^{1}$ pierces $M_{1}^{2} n$ more times in one direction than it does in the other. We shall show that no multiple of $K_{2}^{1}$ bounds in $W^{3}-J$-in fact, not even in $W^{3}$.

Assume $K_{2}^{1}$ is given an orientation so that $m K_{2}^{1}$ bounds in $W^{3}$ and $C_{2}^{2}$ is an oriented complex so that $\partial C_{2}^{2}=m K_{2}^{1}, C_{2}^{2}$ is locally a 2-manifold off $K_{2}^{1}$, and on $K_{2}^{1}$ it locally resembles $m$ sheets with a common edge. Trim $C_{2}^{2}$ back a bit from $K_{2}^{2}$ to obtain an oriented polyhedral 2-manifold $M_{2}^{2}$ (without singularities) so that the piercing number of $\mathrm{Bd} M_{2}^{2}$ and $M_{1}^{2}$ is $m n$. We do not claim that $\mathrm{Bd} M_{2}^{2}$ is connected or even that $M_{2}^{2}$ is connected but we do suppose that $M_{1}^{2}, M_{2}^{2}$ are in such general positions that their intersection is a 1-manifold with boundary along which $M_{1}^{2}$ and $M_{2}^{2}$ locally cross. 
Let $A_{1}^{1}, A_{2}^{1}, \ldots, A_{j}^{1}$ be the components of $M_{1}^{2} \cap M_{2}^{2}$ that are arcs. Remove a tubular neighborhood of $A_{1}^{1}$ from $M_{2}^{2}$ so as to change $M_{2}^{2}$ to an oriented polyhedral 2-manifold with boundary $M_{3}^{2}$. The removal reduces by two the number of points in $M_{1}^{2} \cap \mathrm{Bd} M_{2}^{2}$. However, since the piercing was from different sides, this leaves the piercing number of $\mathrm{Bd} M_{3}^{2}$ and $M_{1}^{2}$ equal to $m n$. By removing tubular neighborhoods about the other $A_{i}^{1}$ 's, we arrive at the contradiction that there is a 2-manifold with boundary $M_{j+2}^{2}$ such that $\mathrm{Bd} M_{j+2}^{2}$ misses $M_{1}^{2}$ but its piercing number with $M_{1}^{2}$ is $m n$. Hence our assumptions that $n K_{1}^{1}$ bounds in $W^{3}-J$ and $m K_{2}^{1}$ in $W^{3}$ cannot both be true.

EXAMPLE. Theorem 8.3 is false if one omits the orientable requirement. Let $P^{2}$ be the projective plane, $J_{0}$ be a simple closed curve in $P^{2}$ such that $P^{2}-J_{0}$ is an open disk, $W^{3}=P^{2} \times(-1,1)$, and $J=J_{0} \times 0$. If $K^{1}$ is any polyhedral simple closed curve in $W^{3}-J, 2 K^{1}$ bounds in $W^{3}-J$.

Definitions. If $B^{3}$ is a polyhedral 3-cell and $J_{1}, J_{2}$ are disjoint simple closed curves in Int $B^{3}$, we say that the (unsigned) linking number of $J_{1}$ and $J_{2}$ is $n$ if $J_{1}$ is homotopic in Int $B^{3}-J_{2}$ to a polyhedral simple closed curve $K_{1}^{1}, K_{1}^{1}$ can be oriented so that it bounds an orientable polyhedral 2-manifold $M^{2}$ in $\operatorname{Int} B^{3}$, and $J_{2}$ is homotopic in Int $B^{3}-K_{1}^{1}$ to a polyhedral simple closed curve $K_{2}^{1}$ such that the piercing number of $K_{2}^{1}$ and $M^{2}$ is $n$. It is known that the linking number of $J_{1}$ and $J_{2}$ is independent of the $K_{1}^{1}, M^{2}, K_{2}^{1}$ selected and is equal to the linking number of $J_{2}$ and $J_{1}$. We say that $J_{1}$ and $J_{2}$ link if this linking number is not 0 . If the linking number is 0 , it is possible to adjust $M^{2}$ so as to get an oriented polyhedral 2-manifold with boundary $M_{1}^{2}$ so that $\mathrm{Bd} M_{1}^{2}=K_{1}^{1}$ and $M_{1}^{2} \cap J_{2}=0$.

THEOREM 8.4. Suppose $B^{3}$ is a polyhedral 3-cell in a polyhedral 3-manifold with or without boundary $N^{3}$ and $C_{1}, C_{2}$ are linking simple curves in Int $B^{3}$. Then if $G$ is the decomposition of $C_{1} \cup C_{2}$ whose only nondegenerate element is $C_{2}$, there is no monotone upper semicontinuous decomposition of $N^{3}$ onto a 3-manifold $W^{3}$ such that the elements of $G$ are elements of the decomposition.

Proof. We show that there is no such decomposition by showing that there is no compact monotone map $g$ of $N^{3}$ onto $W^{3}$ that has elements of $G$ as point inverses. For convenience we suppose $W^{3}$ is polyhedral.

Assume there is such a $g$. Let $U$ be an open subset of $W^{3}$ containing $g\left(C_{1}\right)$ such that $g^{-1}(U) \subset$ Int $B^{3}$ and let $J^{1}$ be a polyhedral simple closed curve in $U-g\left(C_{1}\right)$ such that no multiple of $J^{1}$ bounds in $W-g\left(C_{1}\right)$. That there is such a $J^{1}$ follows from Theorem 8.2.

Let $h$ be a homeomorphism of $J^{1}$ into $g^{-1}(U)-C_{1}$ such that $h\left(J^{1}\right)$ is a polyhedron, and the identity on $J^{1}$ is homotopic to $g h$ in $U-g\left(C_{1}\right)$.

If $h\left(J^{1}\right)$ does not link $C_{1}$, there is an orientation for $h\left(J^{1}\right)$ and an oriented 2complex $C^{2}$ in Int $B^{3}-C_{1}$ such that $\partial C^{2}=h\left(J^{1}\right)$. Then $g\left(C^{2}\right)$ and a singular annulus joining $J^{1}$ and $g h\left(J^{1}\right)$ provides the image of an oriented 2-complex in $M^{3}-g\left(C_{1}\right)$ whose boundary is $J^{1}$. An approximation to this image gives an oriented 
2-complex in $W^{3}-g\left(C_{1}\right)$ whose boundary is $J^{1}$. The case where $h\left(J^{1}\right)$ does not link $C_{1}$ leads to the contradiction that $J^{1}$ bounds in $W^{3}-g\left(C_{1}\right)$.

There remains the case where $h\left(J^{1}\right)$ links $C_{1}$ in Int $B^{3}$. Suppose the linking number is $n$ and that the linking number of $C_{2}$ with $C_{1}$ is $m$. Let $K_{2}^{1}$ be a polyhedral simple closed curve homotopic in Int $B^{3}-C_{1}$ to $C_{2}$. Then $m h\left(J^{1}\right)$ and $n K_{2}^{1}$ each link $C_{1} m n$ times. Hence there are orientations for $h\left(J^{1}\right)$ and $K_{2}^{1}$ and an oriented 2-complex $C_{2}^{2}$ in Int $B^{3}-C_{1}$ such that $\partial C_{2}^{2}=m h\left(J^{1}\right)+n K_{2}^{1}$. An approximation to $m$ singular annuli each joining $J^{1}$ and $g h\left(J^{1}\right), g\left(C_{2}^{2}\right)$, and the image under $g$ of $n$ singular annuli each joining $K_{2}^{1}$ and $C_{2}$ provides us with an oriented 2-complex in $W^{3}-g\left(C_{1}\right)$ whose boundary is $m J^{1}$. The assumption that there was a decomposition of $N^{3}$ into $W^{3}$ having elements of $G$ as elements led to the contradiction that no multiple of $J^{1}$ bounds in $M^{3}-g\left(C_{1}\right)$ but $m J^{1}$ bounds there.

Question. Would Theorem 8.4 be true if instead of saying that $C_{1}$ and $C_{2}$ link in Int $B^{3}$ we required that they be polygonal simple closed curves in Int $B^{3}$ that 1-link there? Recall that two curves 1-link if they do not bound mutually exclusive orientable 2-manifolds.

\section{REFERENCES}

1. R. H. Bing, A characterization of 3-space by partitionings, Trans. Amer. Math. Soc. 70 (1951), 15-27. MR 13, 484.

2. - Concerning hereditarily indecomposable continua, Pacific J. Math. 1 (1951), 43-51. MR 13, 265.

3. - Locally tame sets are tame, Ann. of Math. (2) 59 (1954), 145-158. MR 15, 816.

4. - An alternative proof that 3-manifolds can be triangulated, Ann. of Math. (2) 69 (1959), 37-65. MR 20 \#7269.

5. - "Decompositions of $E^{3}$ " in Topology of 3-manifolds and related topics (Proc. Univ. of Georgia Institute, 1961), Prentice-Hall, Englewood Cliffs, N. J., 1962, pp. 5-21. MR 25 \#4501.

6. B. G. Casler, An imbedding theorem for connected 3-manifolds with boundary, Proc. Amer. Math. Soc. 16 (1965), 559-566. MR 31 \#2730.

7. J. F. P. Hudson and E. C. Zeeman, On regular neighbourhoods, Proc. London Math. Soc. (3) 14 (1964), 719-745. MR 29 \#4063.

8. E. E. Moise, Affine structures in 3-manifolds. V: The triangulation theorem and Hauptvermutung, Ann. of Math. (2) 56 (1952), 96-114. MR 14, 72.

9. - Affine structures in 3-manifolds. VIII: Invariance of the knot-types; local tame imbedding, Ann. of Math. (2) 59 (1954), 159-170. MR 15, 889.

10. J. H. C. Whitehead, Simplicial spaces, nuclei, and m-groups, Proc. London Math. Soc. (2), 45 (1939), 243-327.

11. G. T. Whyburn, "Decomposition spaces" in Topology of 3-manifolds and related topics (Proc. Univ. of Georgia Institute, 1961), Prentice-Hall, Englewood Cliffs, N. J., 1962, pp. 2-4. MR 25 \#4499.

12. E. C. Zeeman, "Polyhedral $n$-manifolds: II: Embeddings" in Topology of 3-manifolds and related topics (Proc. Univ. of Georgia Institute, 1961), Prentice-Hall, Englewood Cliffs, N. J., 1962, pp. 65-70. MR 28 \#1596.

UNIVERSITY OF WISCONSIN

Madison, Wisconsin 53706 